\title{
CARTOGRAFIA PRELIMINAR DOS REGISTROS OFICIAIS DE MAPEAMENTOS DE TERREIROS DE MATRIZES AFRICANAS NO BRASIL
}

\author{
Rafael Sanzio Araújo dos Anjos \\ Richard Denis \\ Raphael Parizotto \\ Estephany Almeida \\ Cleison Ferreira \\ Gustavo Tolentino
}

p. $38-47$

Revista

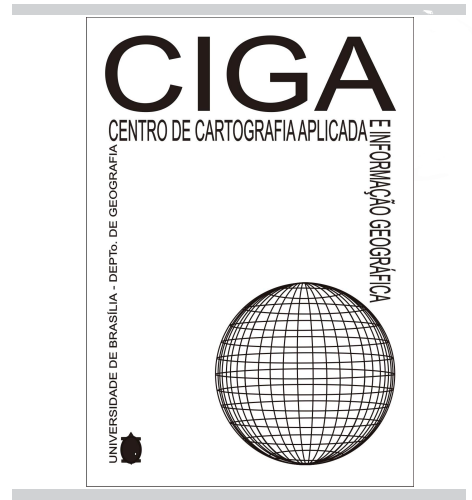

Revista Eletrônica:

Tempo - Técnica - Território,

V.9, N.3 (2018), 38:47

ISSN : 2177-4366
Como citar este ar tigo:

Anjos, R.S.A; Denis, R.; Parizotto, R.; Almeida, E.; Ferreira, C.;

Tolentino, G.; CARTOGRAFIA PRELIMINAR DOS REGISTROS

OFICIAIS DE MAPEAMENTOS DE TERREIROS DE MATRIZES

AFRICANAS NO BRASIL. Revista Eletrônica: Tempo - Técnica Território, v.9, n.3 (2018), p. 38:47 ISSN: 2177-4366.

\section{Disponível em:}

http://periodicos.unb.br/index.php/ciga/issue/view/1429

Este obra está licenciado com uma Licença Creative Commons Atribuição-NãoComercial 4.0 Internacional. 


\section{CARTOGRAFIA PRELIMINAR DOS REGISTROS OFICIAIS DE MAPEAMENTOS DE TERREIROS DE MATRIZES AFRICANAS NO BRASIL}

\section{NOTA TÉCNICA}

\section{Rafael Sanzio Araújo dos Anjos (*), Raphael Parizotto, Estephany Almeida, Cleison Ferreira(**), Richard Denis, Gustavo Tolentino}

(***) E-mail:cartografia@unb.br Site:www.ciga.unb.br

(*) Geógrafo, Doutor em Informações Espaciais Pós-Doutorado Cartografia Étnica. Prof. Titular da Universidade de Brasília / Diretor do Centro de Cartografia Aplicada e Informação Geográfica do Depto. de Geografia - UnB. Coordenador do Projeto do Mapeamento dos Terreiros do DF (Parceria Projeto GEOAFRO-CIGA-UnB-FinatecFCP-MINc (**)Geógrafos Membros da Equipe Técnica do Projeto do Mapeamento dos Terreiros do DF (Parceria Projeto GEOAFRO-CIGA-UnB-Finatec-FCP$\operatorname{MINc}(* * *)$ Estagiários membros da Equipe Técnica do Projeto do Mapeamento dos Terreiros do DF (Parceria Projeto GEOAFRO-CIGA-UnB-Finatec-FCP-MINc)

RESUMO: A partir de um levantamento de dados secundários dos registros dos chamados "mapeamentos dos terreiros" religiosos de matriz africana e afrobrasileira no Brasil, a pesquisa inicial mostrou que existem tipos distintos de trabalhos realizados em algumas unidades políticas do país, mas o problema conceitual verificado (mapa temático, cadastramento detalhado, inventário antropológico-sociológico, listagem com ocorrências, dentre outros produtos), dificulta uma afirmação sobre a realização de fato de uma "cartografia dos terreiros". Esta nota técnica trata da cartografia elaborada para representar estes registros, fundamentais para as políticas públicas reparatórias. O estudo preliminar revela que existem experiências relevantes digitais como analógicas, mas uma investigação mais apurada se faz necessário.

ABSTRACT: From a survey of the records of the so called "terreiros files", the problem of the African matrix and of the company in Brazil, thematic map, detailed registration, anthropological-sociological inventory, occurrence list, among other products) make it more difficult a statement about the realization of a "cartography of the terreiros". This list is elaborated based on a cartography prepared for these records, based on affirmative public policies. The preliminary study is that the evidence is relevant and also similar, but a more accurate investigation becomes necessary.

\section{A CARTOGRAFIA, O ORDENAMENTO DO TERRITÓRIO}

O conhecimento acerca das informações espaciais constitui relevante instrumento para a gestão e planejamento do território, competindo dessa forma, ao 
geografo, a leitura dos elementos espaciais e sua interpretação de forma clara e objetiva.Nesse sentido, as informações geográficas se caracterizam como elementos estratégicos, de modo que a partir dela possibilite responder as diferentes formas e expressões existentes no espaço.

Tendo como ponto de referencia a pesquisa de Mapeamento dos Terreiros de Umbanda e Candomblé do Distrito Federal do Brasil, $1^{\text {a }}$ Etapa - Cartografia Básica (2018), será apresentado os pressupostos instrumentais e metodológicos adotados na produção cartográfica do Mapa de Fluxo dos Terreiros de Umbanda e Candomblé do Distrito Federal para os outros Estados do Território Brasileiro. Com essa produção, busca-se ampliar a contribuição sobre o conhecimento cartográfico, do espaço e suas manifestações no enfoque da pesquisa realizada.

\section{O CIGA}

O Centro de Cartografia Aplicada e Informação Geográfica (CIGA) constitui um importante espaço para o desenvolvimento de atividades, projetos e parcerias dentro da Universidade de Brasília (UnB), oferecendo uma estrutura organizada e equipamentos básicos para o desenvolvimento de estudos relacionados aosdiversos campos de atuação geográfica. O CIGA atua em duas linhas de pesquisa fundamentais, a primeira, a Geografia Afro-Brasileira, e seus segmentos, a segunda, a Instrumentação Geográfica, Educação e Planejamento dos Territórios com seus segmentos. Nesse âmbito, o CIGA, aliado com a cartografia, possibilita tratar e produzir estudos que nos ajudam a compreender as dinâmicas passadas, atuais e futuras atuantes o espaço geográfico.

\section{O MAPA DOS ESTADOS COM REGISTROS OFICIAIS DE MAPEAMENTOS DE TERREIROS DE MATRIZES AFRICANAS NO BRASIL $-2018$}

Em decorrência da pesquisa de Mapeamento dos Terreiros de Umbanda e Candomblé do Distrito Federal surgiu a necessidade de se conhecer onde e em quais estado/municípios no território brasileiro já foram produzidos outros mapeamentos dos terreiros de matrizes africanas.

Objetivando uma leitura simplificada dos mapeamentos e suas características principais, fora elaborado o Mapa dos Estados com Registros Oficiais de Mapeamentos de Terreiros de Matrizes Africanas no Brasil, contudo, foi realizada uma pesquisa a fim 
de se saber quais estados já haviam realizado os mapeamentos, entre eles, que obteve grande destaque foi o Mapeando o Axé, junto a uma parceria com o Ministério do Desenvolvimento Social (MDS); o Mapeamento dos Terreiros de Salvador (CEAO/UFBA) e entre outros realizados.

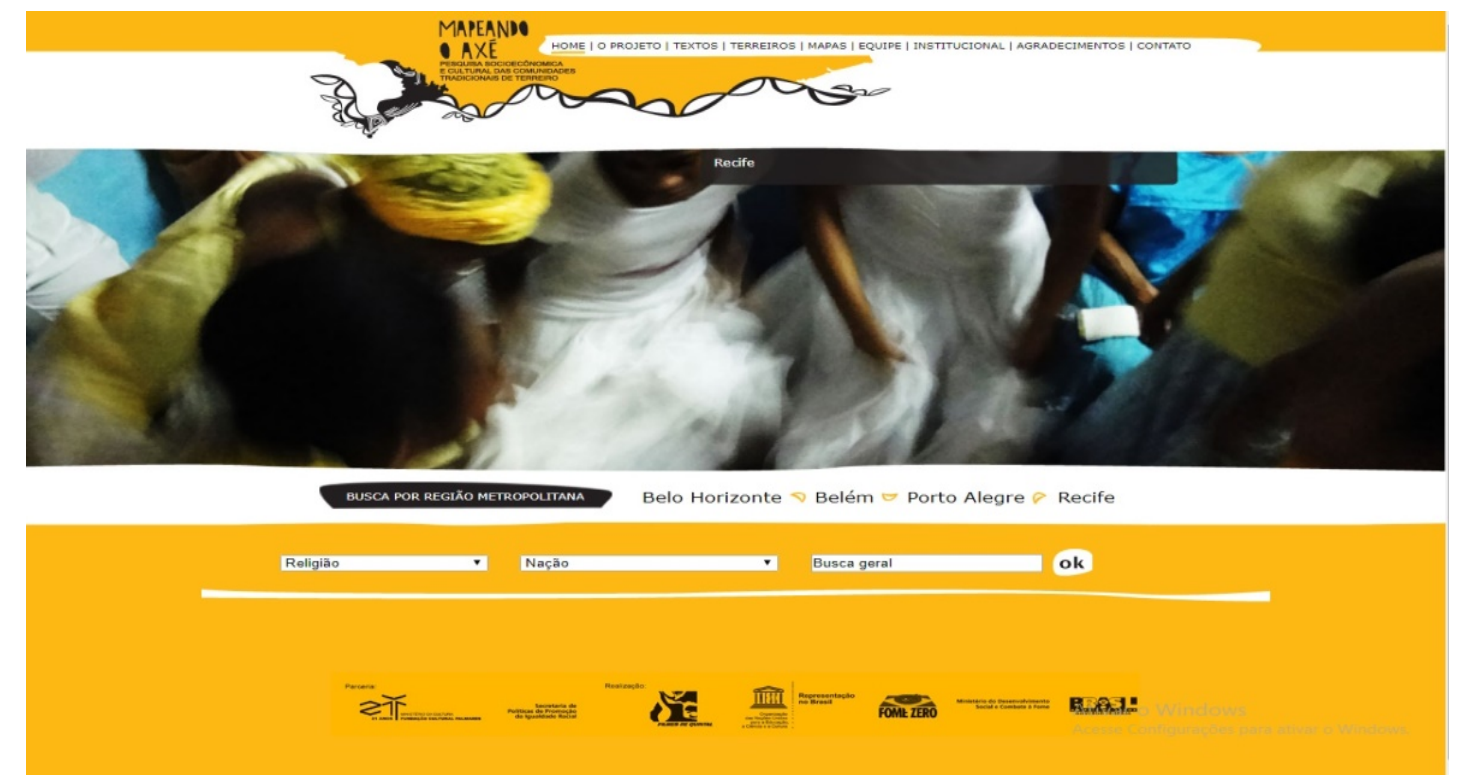

Figura 1. Página do sítio Mapeando o Axé - Ministério do Desenvolvimento Social

Como definição para a inclusão dos mapeamentos, utilizou-se apenas os registros de fontes oficiais, para tanto, o Ministério da Justiça havia disponibilizado arquivo contendo os principais trabalhos de mapeamentos, levantamentos e inventários realizados das comunidades de matrizes africanas. Sendo este documento utilizado como fonte primária das informações presentes no mapa. Além disso, pesquisas na rede de internet foram feitas no intuito de se obter mais informações acerca outros mapeamentos.

Na construção do Mapa, foi utilizada a base cartográfica da América do Sul e do Brasil (CIGA, 2015), os dados dos registros oficiais (estes foram todos organizados em uma planilha, com informações relevantes como: estado, município, fonte da pesquisa e link para o site do mapeamento). 


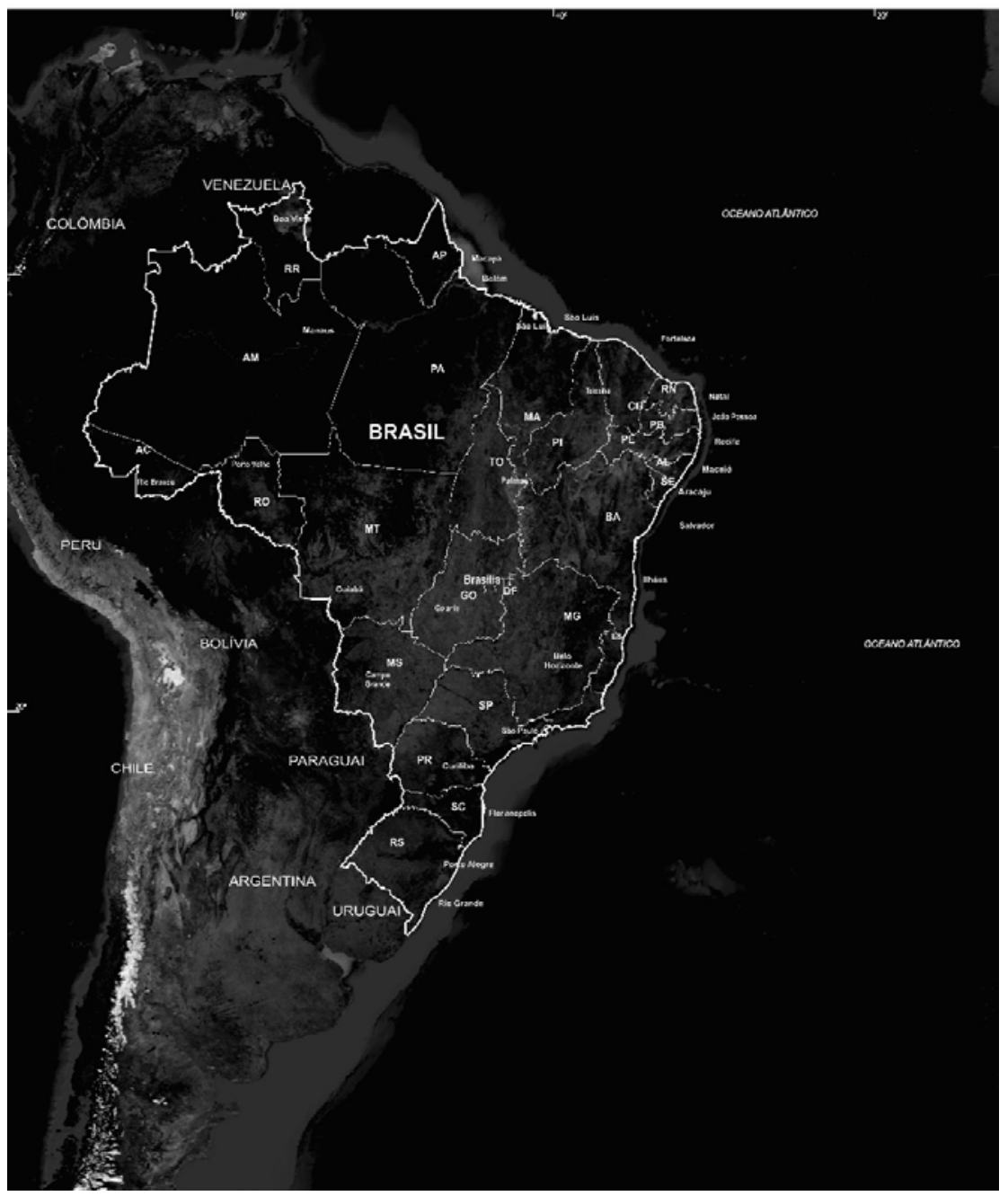

Figura 1. Base cartográfica para o mapa.

Para a manipulação das informações geográficas, foram utilizados os softwares como o CorelDraw e o Microsoft Office Excel, programas que possibilitaram a localização dos pontos, criação dos desenhos e elementos gráficos e organização dos dados em planilhas. A figura 2e 3 expressa as telas de trabalho no CorelDraw e a planilha adotada para controle das informações. 


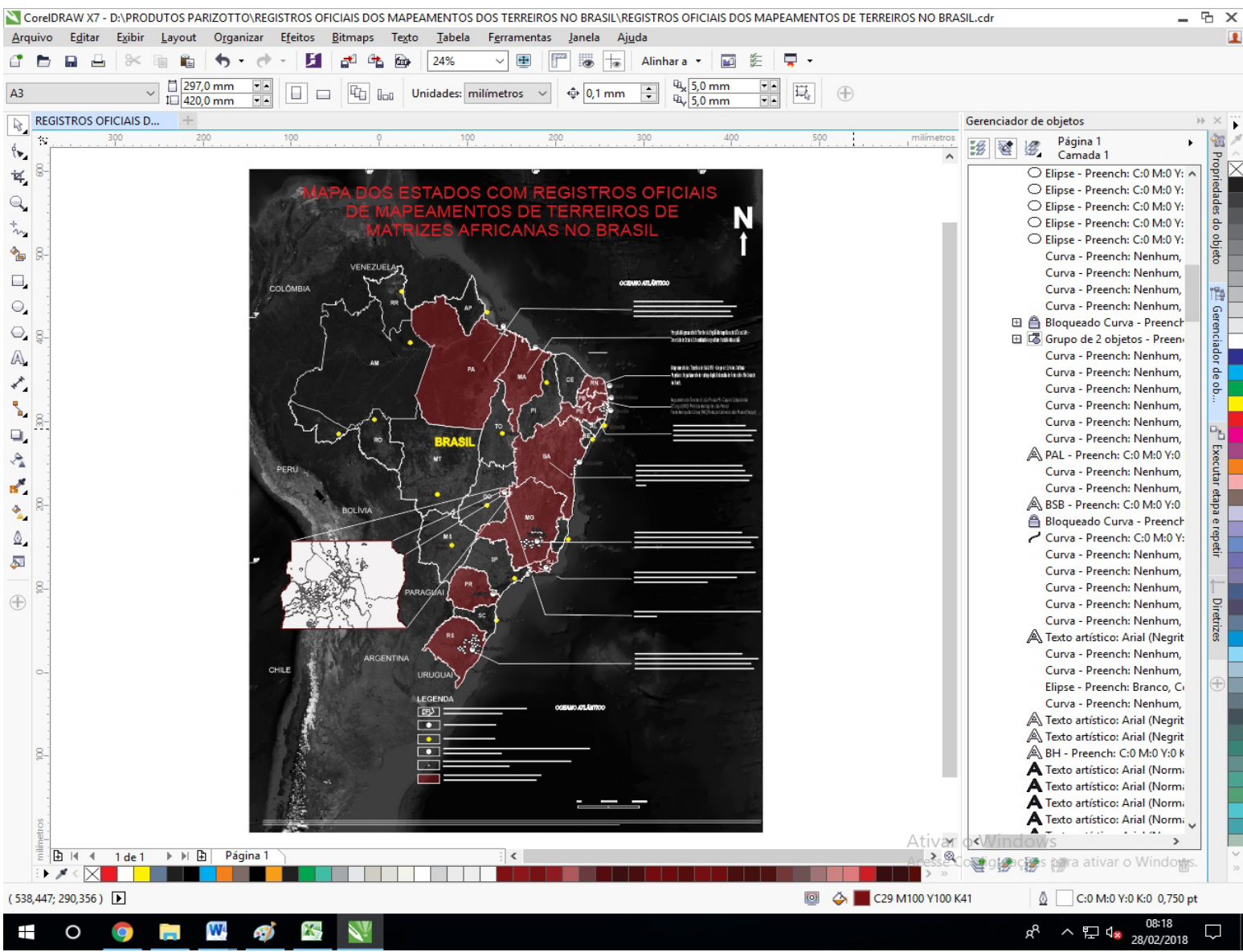

Figura 2. Tela de trabalho no software CorelDraw.



Figura 3. Tela de trabalho no software Microsoft Office Excel. 


\section{PRODUÇÕES CARTOGRÁFICAS}

Para a base cartográfica adotada no mapa, foi utilizada a base cartográfica da América do Sul e do Brasil (CIGA, 2015), abrangendo a região da América do Sul e os Oceanos Atlântico e Pacífico. Com o uso das ferramentas do software CorelDraw foi possível realizar a delimitação dos limites geopolíticos do território brasileiro e seus respectivos estados federados, fator essencial para o destaque posterior dos locais onde ocorreram os mapeamentos.

Além dos limites territoriais, foram adicionados os pontos definidores das capitais de estado e vetorização dos estados em que tiveram registros de mapeamentos das religiões de matrizes africanas. Nas imagens 4 e 5 podem ser observadas partes dos processos de trabalho.
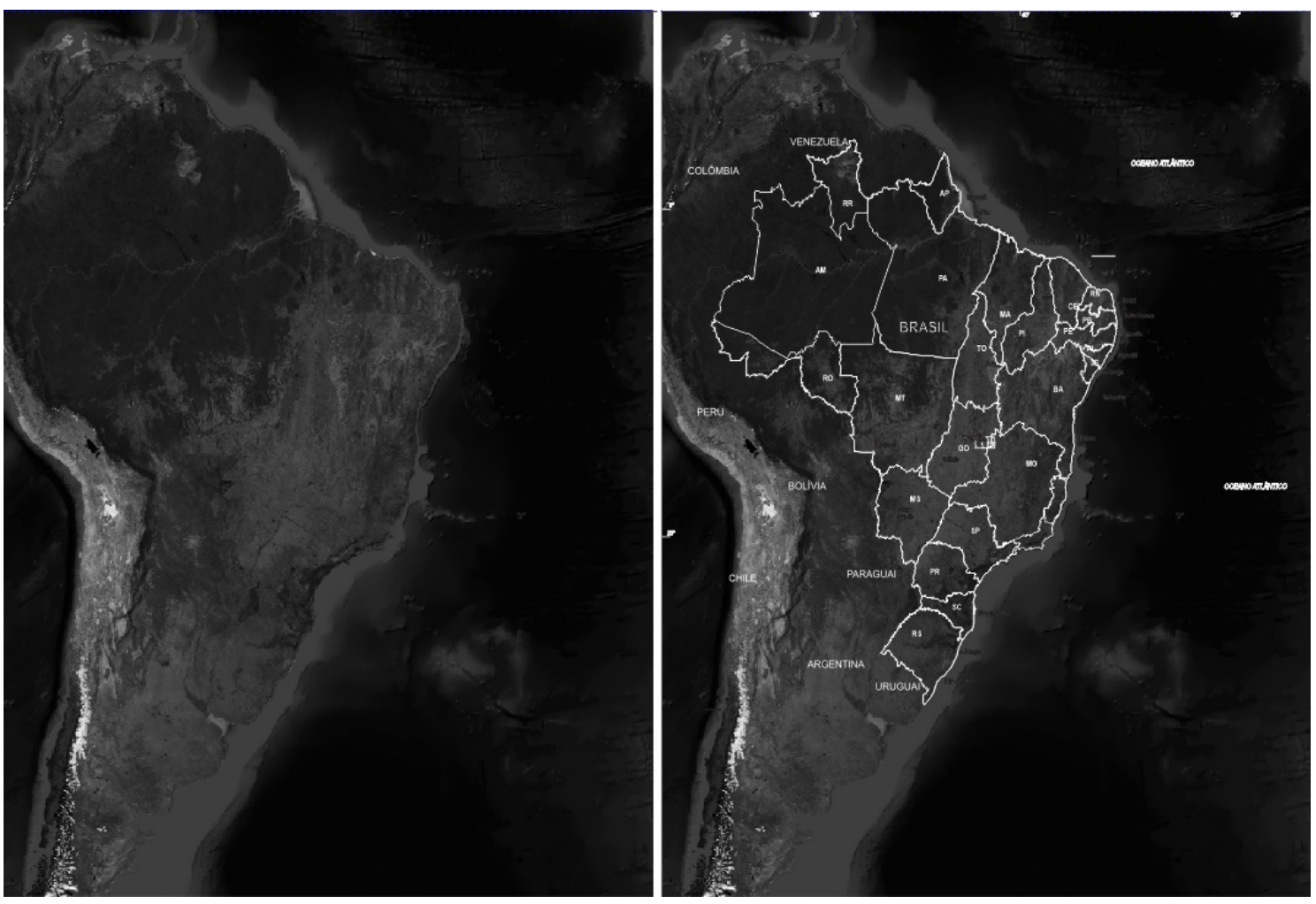

Figura 4. Produção em cima da base cartográfica da América do Sul, foco no Brasil. 


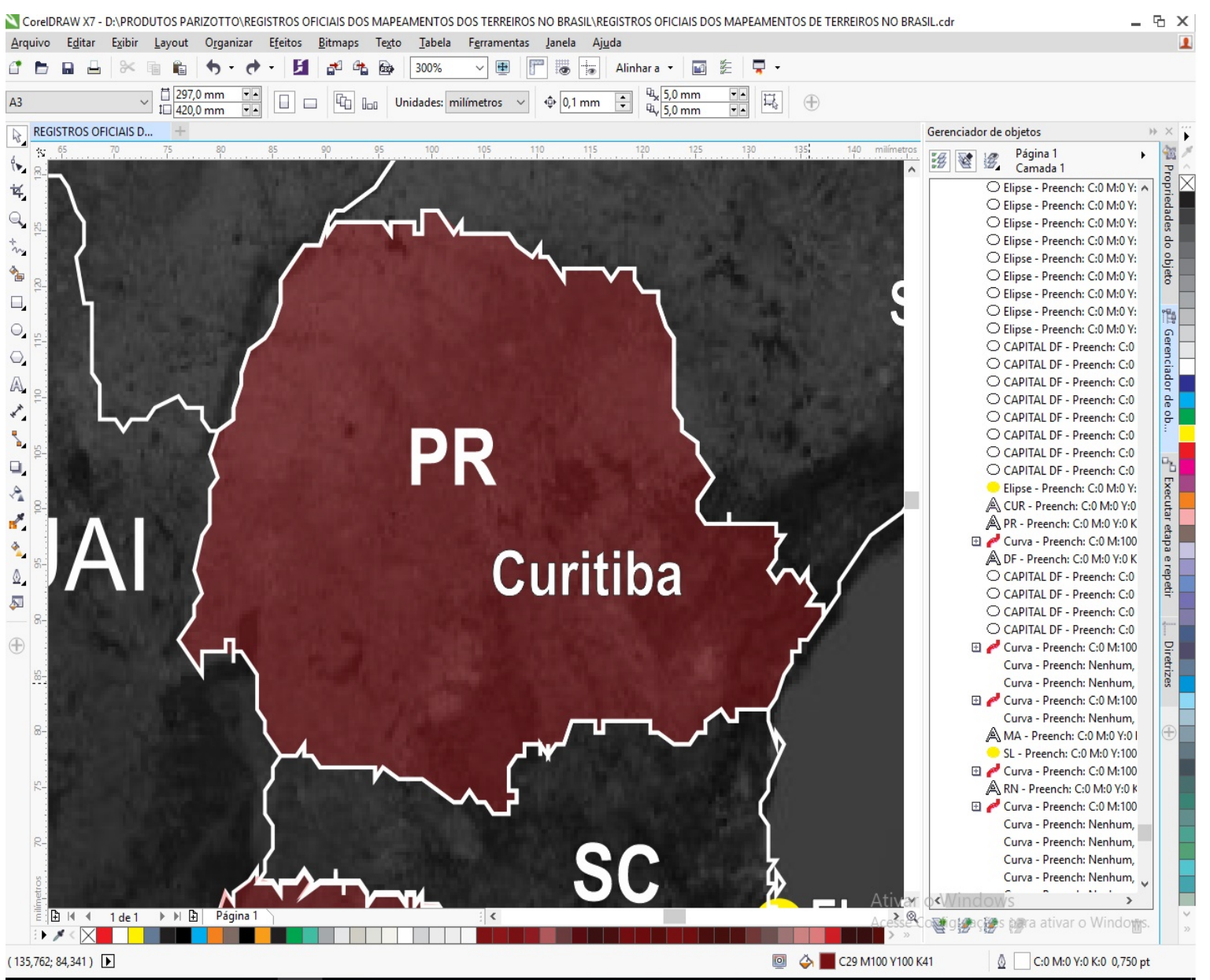

Figura 5. Produção em cima da base cartográfica, delimitação e enfoque nos estado que tiveram mapeamentos realizados.

A etapa seguinte do processo de confecção do mapa se deu com a adição dos pontos de cada município, nessa etapa, para melhor plotagem dos pontos, foi crucial o uso do software Google Earth, no qual possibilitou plotagem aproximada dos pontos com maior precisão no mapa. Além da plotagens dos pontos, fora inseridos uma numeração em cada município/região administrativa, esta numeração, descrita junto à planilha de dados. 


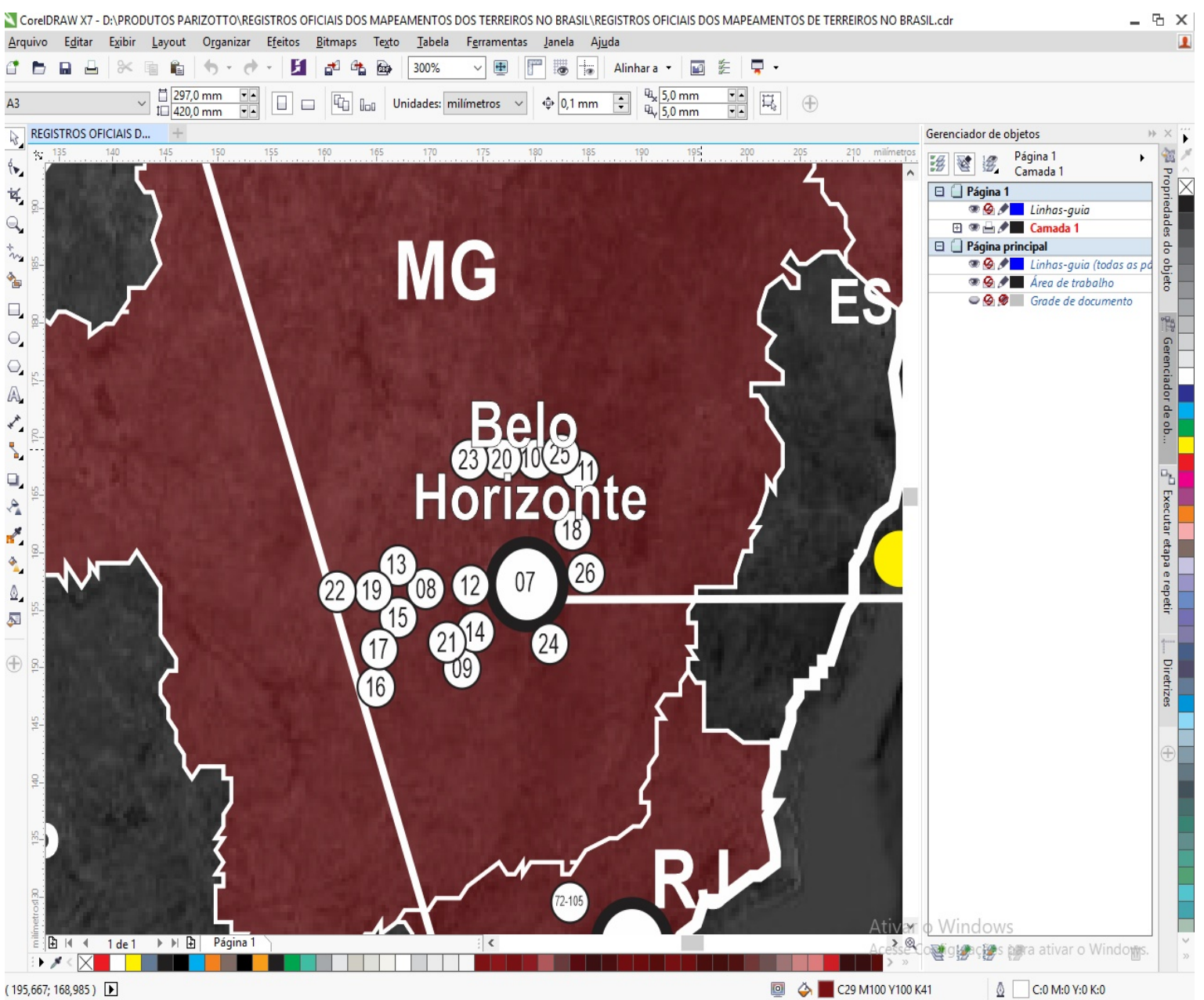

Figura 6. Plotagens dos municípios e regiões administrativas e suas respectivas numerações no mapa.

\section{ELEMENTOS DA LEGENDA}

Para o Mapa dos Estados com Registros de Mapeamentos dos Terreiros no Brasil, em sua legenda são representados itens relevantes para a compreensão e interpretação das informações, entre elas: a divisão política e territorial do Brasil e seus estados federados; ponto da capital política do Brasil; pontos das capitais estaduais brasileiras; ponto das capitais estaduais que tiveram mapeamentos dos terreiros de matrizes africanas; ponto dos municípios que tiveram mapeamentos dos terreiros de matrizes africanas; vetor preenchido dos estados com registro de mapeamento dos terreiros. 


\section{LEGENDA}

DF 3 DIMSÄO TERRITORIAL ATUAL DO BRASIL

ISIGLA DA UNIOAOE POLITICA

\section{OCEANO ATLÂNTICO}

0 CAFITAL POLITICA DO BRASIL

- CAPTAL POLIIICA ESTADUAL

O CAPTAL POLINCA ESTADUAL COM REGISTROS DE MAPEAMENTOS DOS TERREIROS DE MATRIZAFRICANA NO BRASIL

MUNICIPIOS COM REGISTRO OE MAPEAMENTO DOS TERREIROS

DE MATRIZ AFRICANA NO BRASIL

ESTADOS COM REGISTROS OFICIAS DE MAPEAMENTOS

DE TERREIROS DE MATPIZES AFRICANAS NO BRASIL

Figura 7. Categorias de legenda adotadas no mapa.

\section{PRODUTO FINAL}

O produto final do Mapa pode ser visualizado na Figura 8, neste produto ainda fora destacado as referencias e fontes de cada estado que teve realizado algum tipo de mapeamentos dos terreiros de matrizes africanas em seu território. Também foi feito o destaque do Distrito Federal, devido a sua relevância e tamanho na representação cartográfica utilizada. 


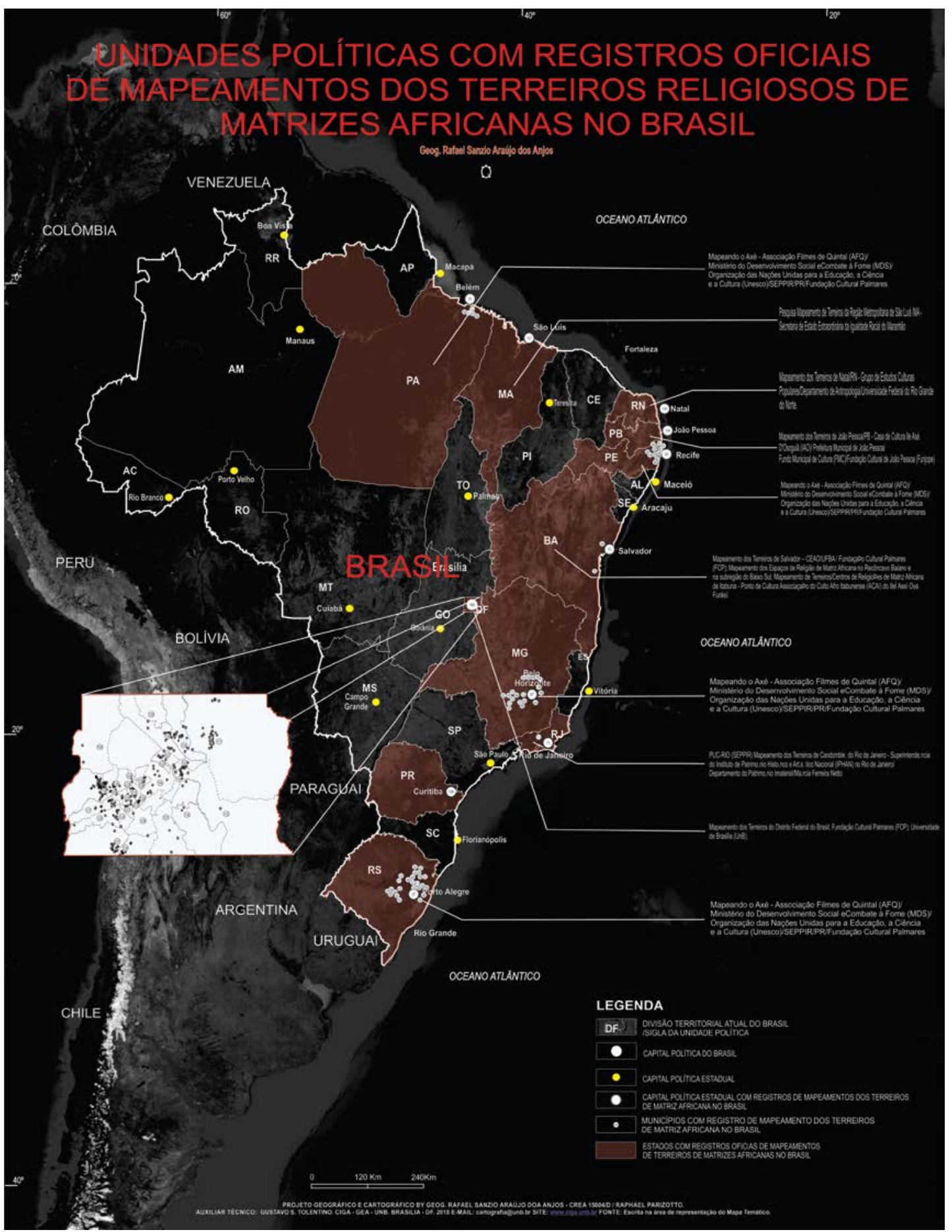

Figura 8. Mapa dos Estados com Registros Oficiais de Mapeamentos de Terreiros de Matrizes Africanas no Brasil.

\section{METATADO ESPACIAL}

\section{BIBLIOGRAFIA}

ANJOS, R.S.A. Mapeamento dos terreiros do Distrito Federal: Cartografia básica 1A. Etapa. Instituto Baobás, Projeto GEOAFRO, CIGA-UnB , Coord. Brasília, DF. 2018 